\title{
Effect of mCIMT with and without interval on hand in patient suffering from stroke
}

\author{
Piyush Bhardwaj, Jaspreet Kaur, Charu Gera, Manoj Malik
}

Department of Physiotherapy, Guru Jambheshwar University of Science and Technology, Hisar, Haryana, India

\begin{abstract}
Introduction. Paresis of hand has severe impact on the life of the stroke patients. Modified constrained induced movement therapy (mCIMT) can be used to treat the involved limb of the patient. It involves constraining of functional hand and increasing the activity of paretic hand which overcomes the "learned disuse" that develops following stroke but the constraining also creates difficulties for the patient and reduces his/her compliance to the treatment. Hence this trial aims to study the effect of $\mathrm{mCIMT}$ with and without interval to see if providing relaxation in between the treatment would be able to produce similar effect as MCIMT without interval protocol.

Matgerial and methods. 44 patients were recruited in the study and were divided in to three groups: $\mathrm{mCIMT}$ without interval $(n=18)$, mCIMT with interval $(n=16)$ and control group $(n=10)$. Participants in three groups were examined for pain and functionality of hand through Patient rated wrist hand evaluation score and Box and Block test at pre and post intervention. The intervention was given for 6 days/week for two weeks. Therapy time was 2 hours and constrain was for 6 hours a day. Clinical trial registration number -CTRI/2019/12/022547.

Results. No baseline differences were found in between the three groups. There was significant increase in box and block test score and significant decrease in PRWHE score in pre and post treatment readings for with and without interval mCIMT but non-significant changes in PRWHE score and box and block test were seen in control group.

Conclusion. Both interval and without interval mCIMT was effective. Interval mCIMT was seen to be more comfortable than without interval mCIMT for the patient.
\end{abstract}

Keywords: modified constrained induced movement therapy, patient rated wrist hand evaluation score, stroke

\begin{abstract}
Abbreviations
mCIMT - modified constrained induced movement therapy;

PRWHE - patient rated wrist hand evaluation score
\end{abstract}

\section{INTRODUCTION}

Stroke is one of the global health problem and leading reason of crippling affliction for adults (1). It is defined as "diminution of brain function by occlusion or bursting of blood vessels in the cerebrum and other parts of central nervous system". The symptoms include one side body weakness, numbness or loss of vision, change in oration and non-orthostatic dizziness (2). One side body weakness is the most common outcome of stroke (3), this affects the functionality of hand which gets more worse within 6 months after stroke due to formation of hand contractures. Patient with stroke decreases the use of affected arm by 80 percent compared to unaffected arm $(4,5)$. Different functional task create mass contraction of different muscles groups in upper limb which is known as synergy (6). It can be functional synergies known as soft synergies which are limited to functional coordination or anatomical synergies also known as hard synergies which are limited to anatomical structures (7). The flexion synergy of upper limb is scapular retraction and elevation, shoulder abduc- 
tion and external rotation, elbow flexion, forearm supination, and wrist and finger flexion (28).

The pervasiveness of stroke has been found to vary from 84 per 100,000 to 262 per $1,00,000$ in rural and from 334 per100,000 to 424 per 100,000 in urban areas in India (stroke fact sheet, India, 2013).The World Health Organisation gave a report that every year 15 million people suffer from stroke, in which mortality rate is 5 million and disability rate is 5 million per 2002 census. Average yearly expenditure on stroke management ranges from $\$ 7,342$ to $\$ 146,149$ in many countries (8).

High blood pressure, high cholesterol, stenosis of carotid arteries, fibrillation in atrium of heart are the major risk factors. Clinically it has been found that treating these risk factors reduce the possibility of stroke to much extent $(4,9)$. Smoking, excessive liquor drinking, resistance for insulin, and high blood sugar are other risk factors. It has also been found that chromosome 12q24.12 near $\mathrm{ALDH} 2$ and its subtypes if gets mutated may cause early onset stroke (10).

Major complications of stroke include Thromboembolic disease, pneumonia, urinary tract infection, bladder dysfunction, bowel dysfunction, pressure sore, shoulder dysfunction, contractures, seizures, fracture and insomnia. These complications can be reduced by proper care and hygiene of the patient (11).

Stroke has huge impact on society. The financial problem has been found between 24 to 33 percent in young patients of stroke (12) as they are dependent on the family (13). Further reducing the work force in the society that affects financially and also psychologically to both patients and their families leading to divorces, separation of families and ultimately decreasing the happiness quotient of the society.

Management includes thrombolytic drugs, antiplatelet drugs, heparin and neuroprotective agents. Surgical intervention is needed in case of increased intracranial pressures for which hemi-craniectomy may be done (14). Clinical rehabilitation concentrates on high intensity, task specific exercises with more and more repetitions of affected hand, which helps in learning function and increasing strength of the affected hand (15). Constrained induced movement therapy (CIMT) is one of the important approaches used clinically to treat patients by constraining the unaffected part of the patient and concentrating on the functions of the affected part using behavioural therapy. CIMT acts by enhancing cortical reorganization and does neuroplastic changes in the motor cortex leading to learning of new tasks and functions(9).

CIMT includes rigorous practice with paretic limb and comprise two parts- restraining of non-paretic extremity during $90 \%$ of waking hours and intensive training of 6 hours or more a day of paretic extremity. However, patient has compliance issue with the CIMT protocol. It has been seen that only $32 \%$ of patients follow CIMT restriction schedule. To deal with this drawback Page and colleagues created a Modified CIMT (mCIMT) protocol that compressed both the vigorous training session of the paretic upper extremity $(30 \mathrm{~min} / \mathrm{d}-2 \mathrm{~h} /$ day) and constrain time of non-paretic upper extremity $(6 \mathrm{~h} / \mathrm{d})(16,17)$

Timings for application for mCIMT are different in literatures. The patient has to be constrained during training as well as at home which becomes difficult for the patient (18). As per longer duration treatment time can decrease the compliance of the patient to the treatment. Hence, a comparative study between the outcomes of constraining patient continuously and with intervals during mCIMT protocol is needed. This helps to modify the mCIMT protocol as per the requirements of the patient. Allowing breaks or intervals in between the treatment and making it more flexible as per the requirement of the patient will help the patient to perform his/her activities of daily living in better way at home and in community.

The rationale of the study was to evaluate the efficacy of mCIMT with or without interval.

\section{MATERIAL AND METHODS}

\section{Design}

This is single blinded randomized controlled trial conducted in two Different Neurological rehab centres at MSS hospital Pitampura Delhi, India and Savitri Devi Goyal Indian stroke and paralysis care centre Nangloi Delhi, India. Stroke survivors coming to OPD and admitted to IPD department of Pitampura and Nangloi centre of Delhi neurological rehab centres were recruited. Potential participants were screened to determine their eligibility. 


\section{Subjects}

Participants were included in the study if the hand was affected and patient was unable to perform Activities of daily living due to stroke, presence of balance and stability in patient, availability of a family member to supervise home exercises was needed.

Participants were excluded from the study -if they have medical problems or cognitive deficit (mini mental status examination score $<24$ ), Intended or actual participation in any other study, Significant pain ( $\geq 4$ in the visual analog scale 17) in any joint, A Botox A injection for the upper extremity within 3 months.

On providing signed informed consent, baseline assessment was done.

\section{Patient recruitment}

50 patients who came to OPD and IPD departments of the rehab centre were screened for the study, 44 met the inclusion criteria and 6 were excluded from the study as they were not comfortable with constrain. The 44 patients were randomly allocated to three groups - without interval mCIMT, with intervals mCIMT and control group (figure 1).

\section{Blinding}

Patients were blinded about treatment assigned to each group. Therapist was aware about the treatment and the patient in each group.

\section{Randomization}

Following study enrolment and completion of the baseline testing, participants were randomised into three groups: mCIMT without interval group, mCIMT with interval group and control group via chit method.

\section{Outcome measures}

Table 1 shows the standard protocol items and outcome measures. Two outcome measures were used box and block test and Patient rated wrist hand evaluation score.

In Box and block test comprise of a wooden box which has 150 blocks in two different compartments are there (figure 2). In which the client is asked to move blocks one after one from one compartment to another within 60 seconds. The test is started with unaffected hand and then affected hand. Additional 15 second trial period can be added. Scoring is done by counting number of blocks transferred by client from one compartment to another in 60 seconds. Scoring of unaffected and affected hand is done separately.

Patient rated wrist hand evaluation score is a 15 -item questionnaire. It comprises of pain and function scores. Pain scoring contains 5 measures rated from 1 to 10 . The maximum score is 50 and minimum is 0 . Function scoring contains 10 measures in total. Maximum score is 50 and minimum is 0 .

The assessment of patient with two outcome measures was done before and after the two weeks of intervention.

\section{Intervention}

Intervention was provided for 6 days a week for two weeks.

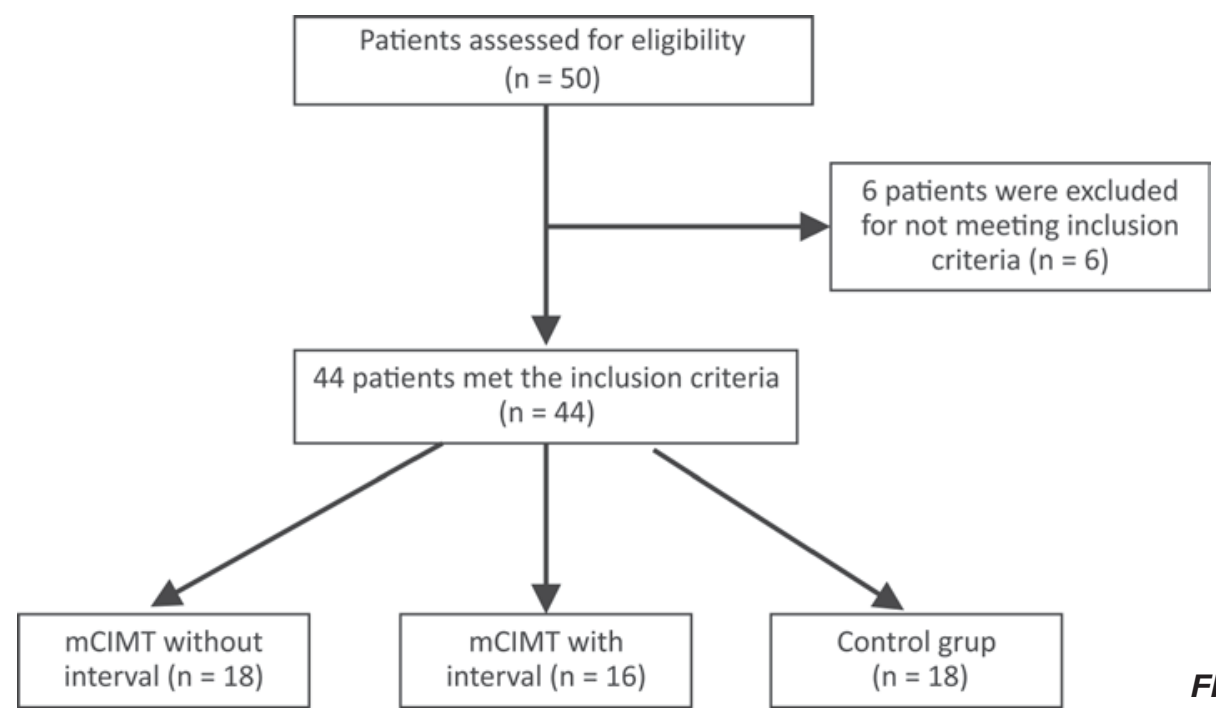


TABLE 1. Standard protocol items

\begin{tabular}{|l|c|c|c|}
\hline \multirow{2}{*}{} & \multicolumn{3}{|c|}{ Study period } \\
\cline { 2 - 4 } & Enrolment & Preallocation & Postallocation \\
\hline Time point & $-\mathrm{t}_{1}$ & $\mathrm{t}_{1}$ & $\mathrm{t}_{2}$ \\
\hline Enrolment & & & \\
\hline eligibility screen & $\mathrm{X}$ & & \\
\hline informed consent & $\mathrm{X}$ & & \\
\hline Randomisation & & $\mathrm{X}$ & \\
\hline Intervention & & & \\
\hline mCIMT without interval & & & $\mathrm{X}$ \\
\hline mCIMT with interval & & & $\mathrm{X}$ \\
\hline Assessment & & $\mathrm{X}$ & \\
\hline box and block test & & $\mathrm{X}$ & \\
\hline PRWHE score & & & \\
\hline
\end{tabular}

$-t_{1}=$ timing before starting of treatment,$t_{1}=$ timing of start of treatment, $t_{2}=$ timing of start of treatment

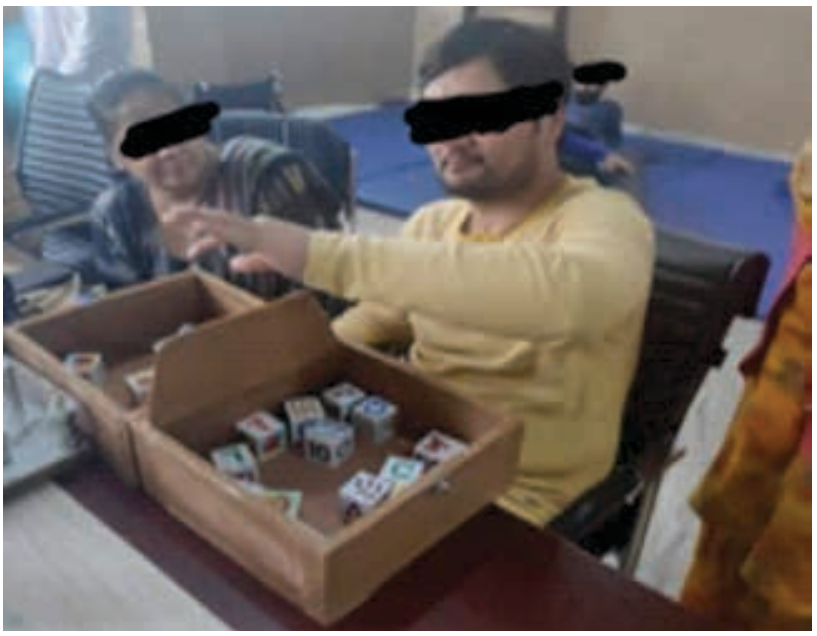

FIGURE 2. Patient performing box and block test

$m$ CIMT without any interval

It comprised of constraining of non-paretic hand for 6 hours (figure 3) continuously which included 2 hours of therapy session too. The therapy session included behavioural therapy according to the patient.

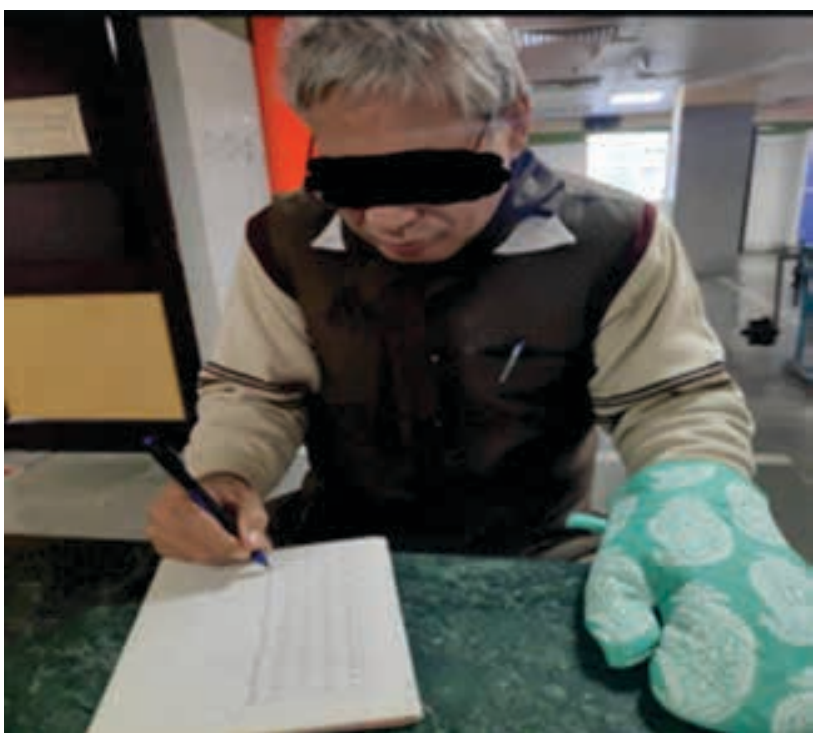

FIGURE 3. Patient is constrained in the left hand and behavioural therapy is applied on right hand
mCIMT with interval

It comprised of constraining the non-paretic hand for 6 hours but with intervals. It included two hours of therapy session with constrain and four hours of constrain with intervals as the convenience of the patient.

\section{Control}

Control group comprised of range of motion exercises which were performed by patient with some stretching for 2 hours during therapy session without any constrain. This therapy was also given 6 days a week for two weeks.

\section{Compliance and safety monitoring}

Participant attendance was recorded. if a participant misses the training they were contacted and reason for their absence was documented. If more than one session was missed a makeup session was scheduled. The study was voluntarily and participant could discontinue if the study on request at any time if there was change in health status (i.e. worsening of function and overall health).

\section{Statistical analysis}

The sample size was calculated using sample size calculation formula below (19):

$$
\mathrm{n}_{\mathrm{i}}=\frac{2\left(\mathrm{Zn}+\mathrm{Z}_{(1-\beta)}\right)^{2} \sigma^{2}}{(\mu \mathrm{s}-\mu \mathrm{t}-\delta)}
$$

Calculation was based on standard deviation $\sigma=$ 8.1 , MCID 3.20 (20) and power of 80 percent and the estimated sample size was 12 .

Statistical analysis was done by SPSS version 26. Baseline characteristics were compared between groups using one-way ANOVA and the Chi- 
squared test. For all outcome measures, intention-to-treat analysis was performed using the last observation carried forward method to account for missing data. The repeated measures of ANOVA were used to assess the intervention effects between groups. Alpha was set at 0.05 , and the $95 \%$ confidence interval (CI) was calculated. If a significant difference was detected, then post hoc tests with the Scheffe method were conducted

\section{RESULTS}

50 patients were selected for this randomised controlled trial. The present study selected subjects from MSS hospital Pitampura Delhi and Savitri Devi Goyal Indian stroke and paralysis care centre Nangloi Delhi. 6 participants did not meet the inclusion criteria and there was no drop out. 44 subjects (18 subjects in the mCIMT without interval, 18 subjects in the mCIMT with interval, and 10 subjects in the control group) participated in this study and finished the protocol completely. The mean age of all participants was $59.08 \pm 9.16$ years old. An aggregate of 29 males and 15 females showed interest in this study. There was no significant difference between the groups in the baseline data ( $p>.05$, as shown in table 2$)$.

\section{Primary outcome measures}

Both Box and block test and PRWHE score were improved significantly more in the intervention groups $(\mathrm{P}=.006) \&(\mathrm{P}=.011)$. As the Mean \pm Standard deviation of box and block test increased from $11.44 \pm 8.27$ to $17.66 \pm 12.03$ in mCIMT without interval group and $9.12 \pm 6.90$ to $15.25 \pm 9.94$ in with interval group with similar decrease in PRWHE score means with $p$ value of 0.00 in both the groups when paired t test was applied (as shown in table 3). While, there was no significant improve- ment in Control group as the mean of box and block test increased only from $9.26 \pm 7.03$ to $10.60 \pm 7.75$ and with similar decrease in PRWHE scores with $\mathrm{p}$ value of 0.00 was seen when paired $t$ test was applied (table 3). Further when all the three groups were compared for Box and block test $(\mathrm{F}(2.46)=10.924, \mathrm{p}=.600)$ and PRWHE score $(\mathrm{F}$ $(2,46)=6.828, p=.003)$ using one-way analysis of variance (ANOVA) no statistical significant difference was found between both interventional groups but there was significant difference when compared to control group (table 4).

For the analysis of the outcome measures, box and block test and PRWHE score, one-way analysis of variance (ANOVA) was used for any statistical significance difference. The between group comparison was done by using ANOVA post Hoc tuckey test. The analysis showed the statistical significant difference in intervention groups as compared to control group. The significance $p$ value of interventional group in box and block is 0.9722 $(>0.05)$ while in control group, it is 0.001 and $0.00(<0.05)$ and similarly in PRWHE group the control group comparison gave the significant value of $0.003(<0.05)$ (as shown in table 5). Hence, there was no statistically significant difference among intervention groups that is MCIMT with interval and without interval although with control group comparison the difference was significant (table 5).

\section{Box and block test}

The two interventional groups, without interval and with interval mCIMT showed significant similar magnitude improvement as compared to control group as seen in graph below. The patient was able to pass more blocks after two weeks in both the groups (Figure 4).

TABLE 2. Table showing baseline characteristics

\begin{tabular}{|c|c|c|c|c|c|}
\hline \multicolumn{2}{|l|}{ Variable } & \multirow{2}{*}{$\begin{array}{c}\text { mCIMT with } \\
\text { interval }\end{array}$} & \multirow{2}{*}{$\begin{array}{c}\begin{array}{c}\text { mCIMT without } \\
\text { interval }\end{array} \\
46.22 \pm 12.48\end{array}$} & \multirow{2}{*}{$\begin{array}{c}\begin{array}{c}\text { Control } \\
\text { Group }\end{array} \\
49.6 \pm 8.8 \\
\end{array}$} & \multirow{3}{*}{$\begin{array}{c}\text { P value } \\
>.05\end{array}$} \\
\hline \multirow[t]{2}{*}{ Age } & Mean \pm SD & & & & \\
\hline & Range & 31 & 54 & 34 & \\
\hline \multirow[t]{2}{*}{ Gender \% } & Male & 63 & 78 & 50 & \multirow[b]{2}{*}{$>.05$} \\
\hline & Female & 37 & 22 & 50 & \\
\hline \multirow{2}{*}{$\begin{array}{l}\text { Pre-intervention data } \\
\text { for PRWHE score }\end{array}$} & Mean $\pm S D$ & $67.37 \pm 22.47$ & $59.66 \pm 22.21$ & $61 \pm 23.97$ & \multirow[b]{2}{*}{$>.05$} \\
\hline & Range & 68 & 69 & 76 & \\
\hline \multirow{2}{*}{$\begin{array}{l}\text { Pre-intervention data } \\
\text { for box and plot test }\end{array}$} & Mean \pm SD & $9.12 \pm 6.90$ & $11.44 \pm 8.27$ & $9.26 \pm 7.03$ & \multirow[b]{2}{*}{$>.05$} \\
\hline & Range & 23 & 25 & 21 & \\
\hline
\end{tabular}

SD-standard deviation 
TABLE 3. Group comparison using paired t test

\begin{tabular}{|l|l|l|l|l|l|l|l|}
\hline Outcome & Group & $\begin{array}{l}\text { Pre-intervention } \\
\text { Mean } \pm \text { SD }\end{array}$ & $\begin{array}{l}\text { Post- intervention } \\
\text { Mean } \pm \text { SD }\end{array}$ & \begin{tabular}{l} 
95\% Confidence interval \\
\cline { 5 - 8 }
\end{tabular} & & t value & P value \\
\hline $\begin{array}{l}\text { Box\& block } \\
\text { test }\end{array}$ & mCIMT without interval & $11.44 \pm 8.27$ & $17.66 \pm 12.03$ & -8.3081 & 4.13629 & 6.293 & 0.00 \\
\hline & & & & & & & \\
\hline & mCIMT with interval & $9.12 \pm 6.90$ & $15.25 \pm 9.94$ & -8.0792 & -4.17077 & -6.68 & 0.00 \\
\hline PRWHE & Control group & $9.26 \pm 7.03$ & $10.60 \pm 7.75$ & -1.8737 & -.79290 & -5.29 & 0.00 \\
\hline & $\begin{array}{l}\text { mCIMT without } \\
\text { interval }\end{array}$ & $59.66 \pm 22.21$ & $50.16 \pm 23.44$ & 5.37009 & 13.62991 & 4.853 & 0.00 \\
\hline & mCIMT with interval & $67.37 \pm 22.47$ & $59.37 \pm 24.64$ & 4.92352 & 11.07648 & 5.543 & 0.00 \\
\hline & Control group & $61.0 \pm 23.97$ & $59.00 \pm 24.33$ & 1.53197 & 2.46803 & 9.165 & 0.00 \\
\hline
\end{tabular}

mCIMT = modified constrained induced movement therapy; SD = standard deviation; PRWHE = patient rated wrist hand evaluation score

TABLE 4. Comparison between group using One-way ANOVA

\begin{tabular}{|l|l|c|c|c|c|}
\hline Outcome & & $\begin{array}{c}\text { Sum of } \\
\text { Squares }\end{array}$ & $\begin{array}{c}\text { Degree of } \\
\text { freedom }\end{array}$ & $\begin{array}{c}\text { Mean } \\
\text { Square }\end{array}$ & F \\
\hline Box \& block test & Between group & 244.214 & 2 & 122.107 & 10.924 \\
\hline & Within group & 514.194 & 46 & 11.178 & \\
\hline & Total & 758.408 & 48 & & \\
\hline $\begin{array}{l}\text { PRWHE } \\
\text { Score }\end{array}$ & Between group & 499.50 & 2 & 249.750 & 6.828 \\
\hline & Within group & 1682.500 & 46 & 36.576 & \\
\hline & Total & 2182.000 & 48 & & \\
\hline
\end{tabular}

$m C I M T=$ modified constrained induced movement therapy; PRWHE = patient rated wrist

hand evaluation score

TABLE 5. Multiple comparison between groups using post hoc Tucke test for box and block test and PRWHE score

\begin{tabular}{|c|c|c|c|c|c|c|}
\hline \multirow[t]{2}{*}{ Variables } & \multirow[t]{2}{*}{ GROUP } & \multirow[t]{2}{*}{ (J) GROUP } & \multirow{2}{*}{$\begin{array}{c}\text { Mean } \\
\text { difference }\end{array}$} & \multirow[t]{2}{*}{ Sig. } & \multicolumn{2}{|c|}{ 95\% Confidence interval } \\
\hline & & & & & Lower bound & Upper bound \\
\hline \multirow{4}{*}{$\begin{array}{l}\text { Box and block } \\
\text { test }\end{array}$} & \multirow{2}{*}{ Without interval } & With interval & .09722 & .996 & -2.6849 & 2.8793 \\
\hline & & Control group & 4.88889 & .000 & 2.0581 & 7.7197 \\
\hline & \multirow{2}{*}{ Control group } & Without interval & -4.88889 & .000 & -7.7197 & -2.0581 \\
\hline & & With interval & -4.79167 & .001 & -7.7017 & -1.8816 \\
\hline \multirow[t]{4}{*}{ PRWHE Score } & Without interval & With interval & 1.50000 & .752 & -3.5325 & 6.5325 \\
\hline & & Control group & 7.50000 & .003 & 2.3794 & 12.6206 \\
\hline & Control group & Without interval & -7.50000 & .003 & -12.6206 & -2.3794 \\
\hline & & With interval & -6.00000 & .022 & -11.2640 & -.7360 \\
\hline
\end{tabular}

$P R W H E=$ patient rated wrist hand evaluation score; sig = significance

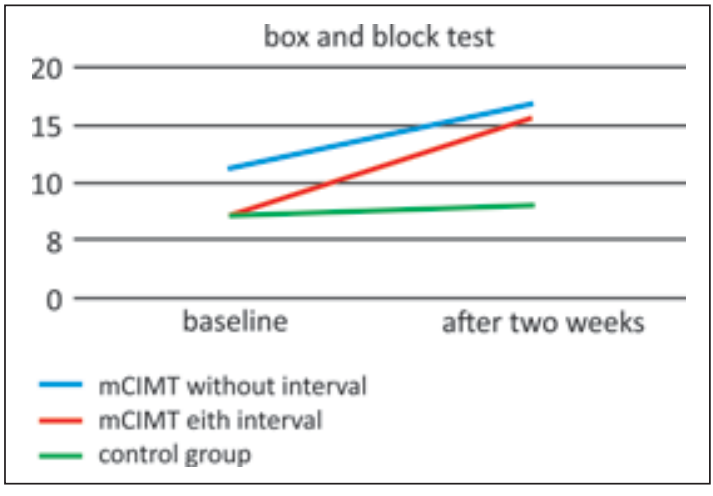

FIGURE 4. Number of blocks transferred in box and block test by patients in three groups

\section{PRWHE SCORE}

The two interventional groups, without interval and with interval mCIMT showed significant sim- ilar magnitude improvement as compared to control group as seen in graph below. The scoring of patient decreased on scale as his pain and morbidity decreased (Figure 5).

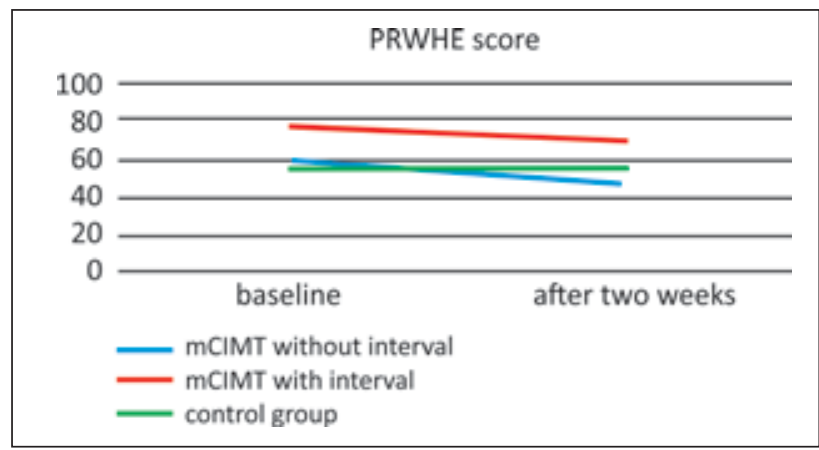

FIGURE 5. PRWHE scoring of patients in three groups 


\section{DISCUSSION}

Prior to this study there was no mCIMT protocol in which intervals were provided to the patient in between the session of mCIMT. These intervals may reduce the difficulty level of the intervention and increase the compliance of the patient. In this RCT patients were actually comfortable with the protocol and showed significant improvement similar to non-interval mCIMT. Practicability, constancy and satisfaction among mCIMT patients were high. Specifically, one mCIMT without interval patient recorded only one incidence of not wearing the sling over the 2 -week period. This information was given by patient's caregivers.

In this study we used two standard clinical tests to assess upper motor functions in patients with stroke that were box and block test and PRWHE score. The results revealed that mCIMT without interval and mCIMT with interval both are equally effective in treating the patient compared to the control group. The past studies never used with interval treatment in mCIMT to treat patients. It was seen that box and block test score was increased from 7 to 16 and patient were able to perform better with hands in mCIMT with interval group. Better performance in box and block test was achieved due to gain of adequate strength and range of motion of hand which not only allowed the patient to transfer but also hold the block for some moments in his fist. Even PRWHE score got decreased from 77 to 70 and improvement was seen in functionality scale and pain scale of the patient. This decrement of PRWHE score was possible due to better adaptation of movements by patient and repetition of more skill-based movement.

The constrained induced therapy protocol is productive but not feasible for some environment, given its exceptional practice and restrain duration. Considerable pilot data suggest that shorter arm practice protocols such as mCIMT is also able to produce cortical reorganization $(21,22)$ and clinically relevant motor changes. Similar to mCIMT without any interval, with interval mCIMT was also able to produce motor changes in the patient due to which all similar effects were seen in the patients.

The mCIMT with and without interval protocol works by decreasing learned non-use (23). The learned non-use happens after an injury which may be due to stroke. After an injury there is decreased CNS and motor activity which increases efforts for the patient while performing movement due to which less movement is produced leading to contraction of cortical representation zone of the part affected. All the unsuccessful attempts by the patient act as punishment in the form of pain or incoordination which leads to behaviour suppression (24) and masked ability of the patient. Lots of compensatory behaviour pattern which are not ideal to perform arrives which allows patient to achieve an action. When these behaviour patterns are repeated, they are reinforced and less effective behaviour gets strengthened. All these things contribute to learn non-use which is reversible in nature (23).

The mCIMT with interval decreased the learned nonuse by increasing motivation of the patient to perform the task. It increased the affected limb use leading to its positive reinforcement. Further practice and reinforcement lead to use dependent cortical reorganization. Cortical representation occurs of the more affected arm, which provides the neural basis for a permanent increase in the use of the extremity. This leads to reversal in learned nonuse and limb use in permanent life situation. And was the main reason for improvement seen in patients (23).

It was observed that mCIMT with interval protocol was more comfortable for the patient compared to without interval mCIMT and compliance issue was nominal. As two weeks was minimal time to see the effect in stroke cases the effect of mCIMT with interval were not that dramatic. If the timing was more the result of outcome measures could have been much better. This protocol was more feasible for females who were house wives and had to take care of kids and husbands at home which required use of unaffected limb for required time. This protocol worked nicely over young age patients who had coordination issues with fingers. It was seen this protocol can help the patient to improve gripping and writing.

The finding of box and block test in our intervention was consistent (25) in which two weeks protocol was followed and improvement of 9 to 16 blocks was seen. Similarly in other neurological condition also Box and block test values was consistent with our study in which the mean value of blocks increases from 2.3 to 4.7 (26). PRWHE 
score was never used before in any study and its results also came promising in our study. Our study results provided more insight over easing the treatment timing which could benefit the patient similarly and would create less compliance issues.

First limitation was the small study sample was used in the study to evaluate the benefit of MCIMT without interval protocol which doesn't give clear picture of the generalizability of the outcomes and preclude meaningful analyses of the effects of participant characteristics, such as gender or side of stroke, on treatment outcomes.

Second limitation was the assessment of outcomes was not blinded.

Third limitation was timing taken of two weeks was less in this protocol to see the proper effect of intervention over patient.

Fourth limitation was due to constrain of timing and hit of Corona epidemic the control group didn't get desired sample size of patients.

Fifth limitation was the therapy protocol is more concentrated on hand movements rather than on elbow and shoulder.

\section{REFERENCES}

1. Emergency and comprehensive care for stroke needed. Lancet. 2009 May 2;373(9674):1496.

2. Hankey GJ, Blacker DJ. Is it a stroke? BMJ. 2015;350(January):1-6.

3. Wolfe CDA. The impact of stroke. Br Med Bull. 2000;56(2):275-86.

4. Xie X, Atkins E, Lv J, Bennett A, Neal B, Ninomiya T, et al. Effects of intensive blood pressure lowering on cardiovascular and renal outcomes: Updated systematic review and meta-analysis. Lancet. 2016;387(10017):435-43.

5. Strømmen AM, Christensen T, Jensen K. Quantitative measurement of physical activity in acute ischemic stroke and transient ischemic attack. Stroke. 2014;45(12):3649-55.

6. Shim JK, Park J, Zatsiorsky VM, Latash ML. Adjustments of prehension synergies in response to self-triggered and experimenter-triggered load and torque perturbations. Exp Brain Res. 2006;175(4):641-53.

7. McMorland AJC, Runnalls KD, Byblow WD. A neuroanatomical framework for upper limb synergies after stroke. Front Hum Neurosci. 2015;9(FEB):1-6.

8. Luengo-Fernandez R, Gray AM, Rothwell PM. Costs of stroke using patient-level data: a critical review of the literature. Stroke. 2009;40(2):18-23.

9. Collins R, Reith C, Emberson J, Armitage J, Baigent C, Blackwell L, et al. Interpretation of the evidence for the efficacy and safety of statin therapy. Lancet. 2016;388(10059):2532-61.

10. Habibi-Koolaee M, Shahmoradi L, Niakan Kalhori SR, Ghannadan $\mathrm{H}$, Younesi E. Prevalence of Stroke Risk Factors and Their Distribution Based on Stroke Subtypes in Gorgan: A Retrospective Hospital-Based Study - 2015-2016. Neurol Res Int. 2018;2018.

11. Kersten P, Low JTS, Ashburn A, George SL, McLellan DL. The unmet needs of young people who have had a stroke: Results of a national UK survey. Disabil Rehabil. 2002;24(16):860-6.

12. Rand D, Eng JJ. Disparity between functional recovery and daily use of the upper and lower extremities during subacute stroke rehabilitation. Neurorehabil Neural Repair. 2012;26(1):76-84.
New and upcoming mCIMT interventions need to take in evaluation the effect of internet bases and offline based mCIMT protocols in real world setting. Few evidence (27) suggest that only 15 minutes of task specific work can produce desired cortical organization and can induce motor learning hence a study must be done to find the exact dosage of therapy session which can produce maximum effect in the patient. The timing given to mCIMT without interval can be increased further to see more better results.

\section{CONCLUSIONS}

After 2-weeks of with and without interval mCIMT protocol, the functional ability of patients with stroke improved significantly in both groups. However there was non-significant difference in between-group comparison. This study concluded that it was more convenient to use with interval mCIMT.

13. Low JTS, Kersen P, Ashburn A, George S, McLellan DL. A study to evaluate the met and unmet needs of members belonging to young stroke groups affiliated with the stroke association. Disabil Rehabil. 2003;25(18):1052-6.

14. Tierney WS, Provencio JJ. Medical management of stroke. Handb Clin Nutr Stroke. 2013;70(Suppl I):139-52.

15. Siegert RJ, Lord S, Porter K. Constraint-induced movement therapy: Time for a little restraint? Clin Rehabil. 2004;18(1):110-4.

16. Page SJ, Sisto S, Levine P, Johnston M V., Hughes M. Modified constraint induced therapy: A randomized feasibility and efficacy study. J Rehabil Res Dev. 2001;38(5):583-90.

17. Page SJ, Levine P, Leonard A, Szaflarski JP, Kissela BM. Modified Constraint-Induced Therapy in Chronic Stroke: Results of a Single-Blinded Randomized Controlled Trial. Phys Ther. 2008; 88(3):333-40.

18. Alexandre NMC, Nordin M, Hiebert R, Campello M. Predictors of compliance with short-term treatment among patients with back pain. Rev Panam Salud Publica/Pan Am J Public Heal. 2002;12(2):86-94.

19. Charan J, Biswas T. How to calculate sample size for different study designs in medical research? Indian J Psychol Med. 2013; 35(2):121-6.

20. Wang TN, Liang KJ, Liu YC, Shieh JY, Chen HL. Psychometric and Clinimetric Properties of the Melbourne Assessment 2 in Children With Cerebral Palsy. Arch Phys Med Rehabil. 2017;98(9):1836-41.

21. Yu C, Wang W, Zhang Y, Wang Y, Hou W, Liu S, et al. The effects of modified constraint-induced movement therapy in acute subcortical cerebral infarction. Front Hum Neurosci. 2017;11(May):1-9.

22. Szaflarski JP, Page SJ, Kissela BM, Lee JH, Levine P, Strakowski SM. Cortical Reorganization Following Modified Constraint-Induced Movement Therapy: A Study of 4 Patients With Chronic Stroke. Arch Phys Med Rehabil. 2006;87(8):1052-8.

23. Taub E. The behavior-analytic origins of constraint-induced movement therapy: An example of behavioral neurorehabilitation. Behav Anal. 2012;35(2):155-78. 
24. Holth P. Two definitions of punishment. Behav Anal Today. 2005; 6(1):43-7.

25. Siebers A, Öberg U, Skargren E. The effect of modified constraintinduced movement therapy on spasticity and motor function of the affected arm in patients with chronic stroke. Physiother Canada. 2010;62(4):388-96.

26. Nagarani S. Effects of Modified Constrained Induced Movement Therapy to Improve the Upper Limb Functional Activities and Gross Manual Dexterity on Hemiparetic Cerebral Palsy Children. Int J Neurorehabilitation. 2015;02(03):2-4.

\section{Conflict of interest: none declared}

Financial support: none declared
27. Classen J, Liepert J, Wise SP, Hallett M, Cohen LG. Rapid plasticity of human cortical movement representation induced by practice. J Neurophysiol. 1998;79(2):1117-23.

28. O'Sullivan SB. Strategies to Improve Motor Function. In: Physical Rehabilitation 6th ed. O'Sullivan SB, Schmitz TJ, Fulk GD (eds.). McGraw Hill Medical, 2007. 